\title{
The Research on the Nonlinear Torsion Movement of Three Wire Pendulum
}

\author{
Yan Huang ${ }^{1 \mathrm{a}}$, Hong $\mathrm{Dai}^{1 \mathrm{~b}}$ and Songlin $\mathrm{He}^{2 \mathrm{c}}$ \\ ${ }^{1}$ Department of physics science and technology, Kunming University, Kunming, 650214, China \\ ${ }^{2}$ Department of asset and laboratory management, Kunming University, Kunming, 650214, China

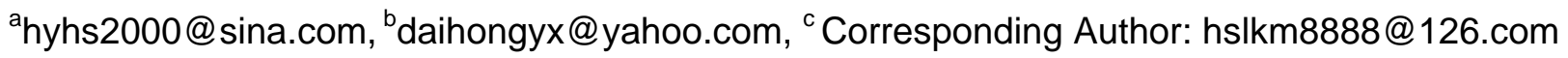

Keywords: three wire pendulum; nonlinear vibration; period

\begin{abstract}
The nonlinear torsion vibration of the three wire pendulum has been studied in this paper. The differential equation of movement of suspension disc has been obtained by theoretical analysis. The vibration curve and phase diagram have been gained by solving the differential equation numerically. It is found that vibration properties change with the difference of system parameters. Theoretical analysis gives the formula of vibration period of the long cycloid three-wire pendulum. The effectiveness of this formula is verified the experiment.
\end{abstract}

\section{Introduction}

Using three wire pendulum to measure the moment of inertia of rigid body is a very common method in physics and engineering practice $[1,2]$. In this method, the long wire pendulum was used and the amplitude of torsion angle is limited less than $5^{\mathrm{O}}$ and the torsion movement of three wire pendulum is thought of as harmonic vibration. But when the angle amplitude is much larger than $5^{\circ}$, or if the length of wire of pendulum is same as or shorter than the radius of suspension disc, the nonlinearity of vibration is not neglected. In this case, there are some questions to be answered. Such as how does the suspension disc move, how does the period of vibration change with the amplitude of torsion angle, and so on. In this paper, we try to answer these questions.

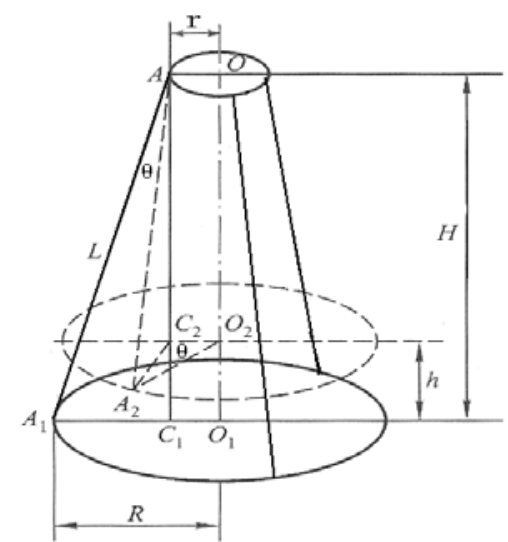

Fig. 1 The schematic of three wire pendulum

\section{The differential equation of the torsion motion of the suspension disc}

Three wire pendulum is composed of two parallel discs which are connected by three isometric suspension wires distributed symmetrically. The upper and the lower circular discs are called the suspension disc and pad disc respectively. As shown in Fig.1, the radius of upper and lower disc are marked as $r$ and $R$ respectively. The length of the suspension line is $L$. Two discs are adjusted horizontally. The distance of two discs is $H$. The centers of two discs are in the same vertical line $O_{1} O_{2}$, and the suspension disc can move around $O_{1} O_{2}$ reversely. Assuming the wire length 
unchanged when the suspension disc reversing angle $\theta$, it will rise $h$. In this case, the geometric constraint relation is given as

$2 H h-h^{2}=2 \operatorname{Rr}(1-\cos \theta)$.

In practical experiment, $h<<H$, thus, if ignored $h^{2}$, above equation becomes

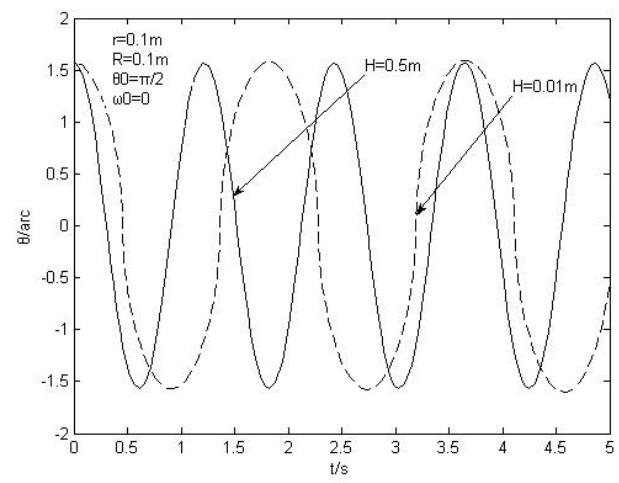

Fig. 1 The schematic of three wire pendulum schematic

$h=\frac{R r}{H}(1-\cos \theta)$.

Taking Eq. 2 derivate to t, the velocity of suspension disc translating up and down can be gained as

$v=\frac{R r}{H} \sin \theta \dot{\theta}$

If the influence of the friction and resistance is ignored, the mechanical energy of the three wire pendulum system is conservative when the suspension disc moves reversely. By the law of conservation of mechanical energy, we obtain

$$
\frac{1}{2} I \dot{\theta}^{2}+\frac{1}{2} m\left(\frac{R r}{H} \sin \theta \dot{\theta}\right)^{2}+\frac{m g R r}{H}(1-\cos \theta)=\frac{m g R r}{H}\left(1-\cos \theta_{0}\right) .
$$

Where $m$ and $I$ are mass and the moment of inertia of the suspension disc respectively. The $\theta_{0}$ is the largest torsion angle of suspension disc.

Taking Eq. 4 derivate to t, the differential equation of the movement of suspension disc can be gained as 


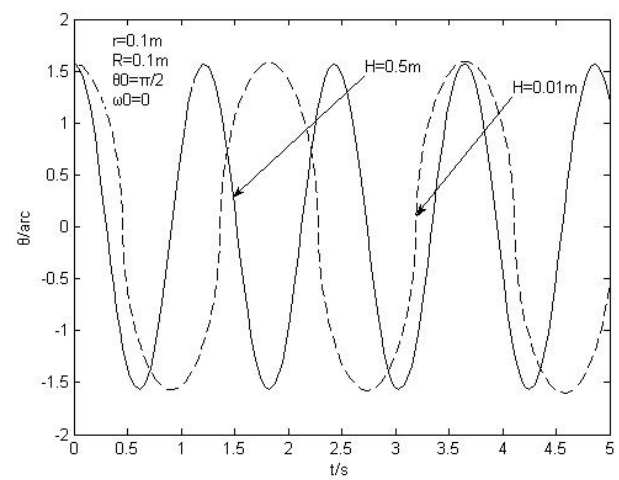

Fig.2 The vibration curve

$$
\left(I+\frac{m R^{2} r^{2}}{H^{2}} \sin ^{2} \theta\right) \ddot{\theta}+\frac{m R^{2} r^{2}}{2 H^{2}} \sin 2 \theta \dot{\theta}^{2}+\frac{m g R r}{H} \sin \theta=0
$$

\section{No-load suspension disc vibration curve and phase diagram}

In a common way, the suspension disc is homogeneous disc made of the metal material. If it is no-load, its moment of inertia can be expressed as $I=\frac{1}{2} m R^{2}$. Thus Eq. 5 is simplified as

$$
\left(\frac{1}{2}+\frac{r^{2}}{H^{2}} \sin ^{2} \theta\right) \ddot{\theta}+\frac{r^{2}}{2 H^{2}} \sin 2 \theta \dot{\theta}^{2}+\frac{g r}{H R} \sin \theta=0 .
$$

Eq.6 is nonlinear differential equation, [3,4] using Runge-Kutta method to solve it numerically [5], the vibration curve and phase diagram can be obtained as Fig. 2 and 3.

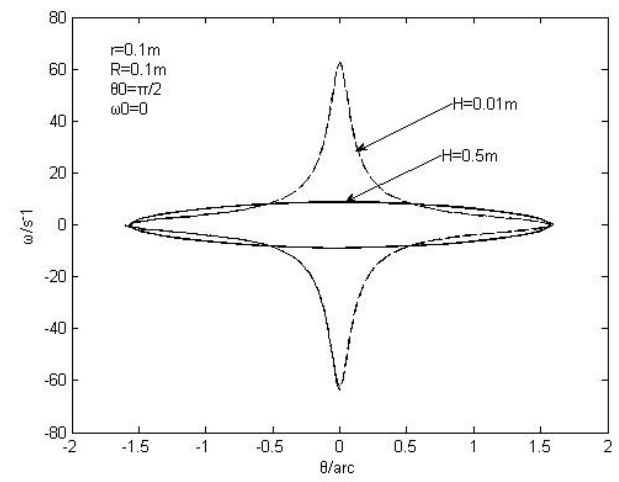

Fig.3 The phase diagram

The Fig. 2 and 3 show that the vibration curve is more sinusoid and the phase diagram is more like ellipse while $H$ is larger. These reveal that the movement of the long wire pendulum is like harmonic vibration but short wire pendulum is not.

\section{The period of vibration of suspension disc}

According to Eq. 5, we gain that

$$
\dot{\theta}^{2}=\frac{2 m g R r\left(\cos \theta-\cos \theta_{0}\right)}{H\left(I+\frac{m R^{2} r^{2} \sin ^{2} \theta}{H^{2}}\right)} .
$$




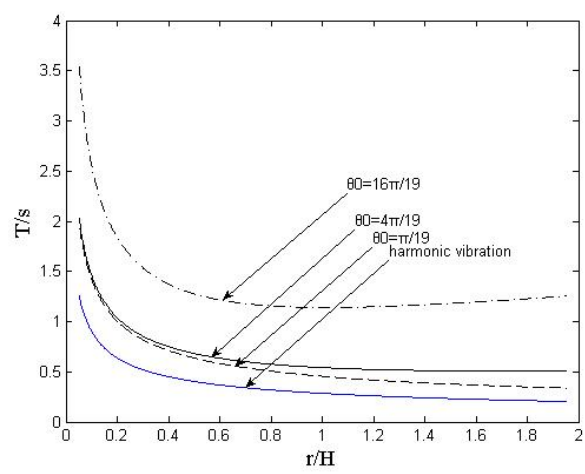

Fig.4 The relation of period varying with the $\mathrm{r} / \mathrm{H}$ and $\theta_{0}$

For no-load suspension disc, $I=\frac{1}{2} m R^{2}$, then we obtain from above equation

$$
d t=\left[\frac{2 g r\left(\cos \theta-\cos \theta_{0}\right)}{H R\left(\frac{1}{2}+\frac{r^{2} \sin ^{2} \theta}{H^{2}}\right)}\right]^{-\frac{1}{2}} d \theta .
$$

Taking integral of above equation, considering that the torsion angle changes from 0 to $\theta_{0}$ during a quarter of period, the period expression relation can be gotten as

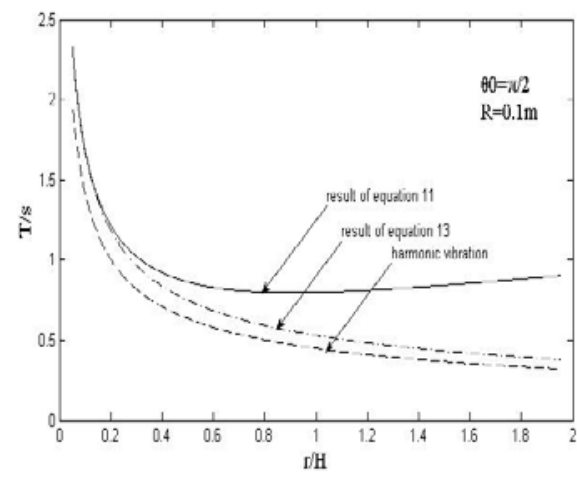

Fig. 5 The comparison of different period expression

expressionexpexpressionschem

$$
T=4 \int_{0}^{\theta_{0}}\left[\frac{2 g r\left(\cos \theta-\cos \theta_{0}\right)}{H R\left(\frac{1}{2}+\frac{r^{2}}{H^{2}} \sin ^{2} \theta\right)}\right]^{-\frac{1}{2}} d \theta .
$$

To observe the vibration period varying with the system and state factors, taking integral to Eq.9 numerically and drawing a picture. Fig. 4 is a result of $R=0.1 \mathrm{~m}$. It is shown that the period is increased with the increasing of angle amplitude $\theta_{0}$ for same pendulum. The smaller $\theta_{0}$ is, the vibration period is closer to the result of harmonic vibration.

In practical experiment, the $\mathrm{r}$ is much less than $\mathrm{H}$, namely, $\frac{r^{2}}{H^{2}} \rightarrow 0$, thus Eq.11 becomes 


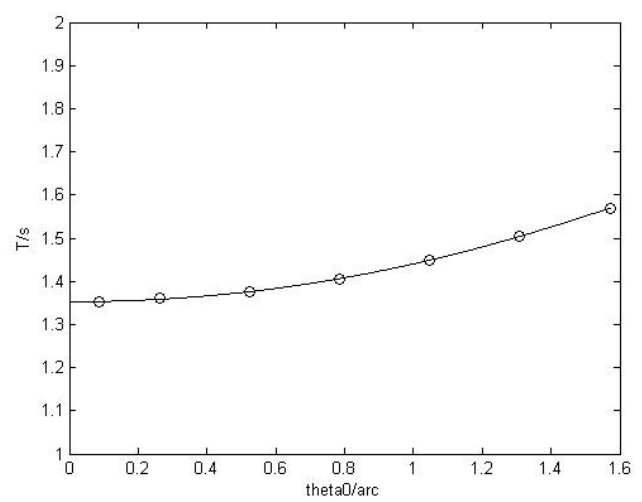

Fig. 6 The experiment data and result of Eq.15

$$
T^{\prime}=2 \int_{0}^{\theta_{0}}\left[\frac{g r\left(\cos \theta-\cos \theta_{0}\right)}{H R}\right]^{-\frac{1}{2}} d \theta .
$$

Taking variable substitution $\sin \frac{\theta}{2}=\sin \frac{\theta_{0}}{2} \sin \varphi \quad\left(0 \leq \varphi \leq \frac{\pi}{2}\right)$,then

$$
T^{\prime}=4 \sqrt{\frac{H R}{2 g r}} \int_{0}^{\frac{\pi}{2}} \frac{d \varphi}{\sqrt{1-\sin ^{2} \frac{\theta_{0}}{2} \sin ^{2} \varphi}} .
$$

The integral of above equation is first type elliptic integral. While marking harmonic vibration period

$$
T_{0}=\sqrt{2} \pi \sqrt{\frac{H R}{g r}},
$$

the period of long wire pendulum can be expressed as

$$
T^{\prime}=T_{0}\left[1+\frac{1}{4} \sin ^{2} \frac{\theta_{0}}{2}+\frac{9}{64} \sin ^{4} \frac{\theta_{0}}{2}+\ldots\right] \text {. }
$$

Fig. 5 shows the results of Eq.9, 13 and harmonic vibration. It reveals that, when $\frac{r}{H} \leq 0.2$, the result of Eq.9 is very close to the result of Eq.13. This suggests that if $\frac{r}{H} \leq 0.2$, the pendulum is considered as long wire pendulum, and its vibration period can be calculated by Eq.13.

\section{The experiment examination}

To examine the correction of above statement, we did the practical experiment in mech anics laboratory. The experiment process on the tester (DH4601A )of Moment of inertia wh ich is made by Hangzhou Dahua instrument manufacturing co., LTD. The system factors ar e $r=4.332 \pm 0.002 \mathrm{~cm}, R=9.180 \pm 0.002 \mathrm{~cm}, H=42.75 \pm 0.05 \mathrm{~cm}, m=1205.00 \pm 0.01 \mathrm{~g}$. We me asure the time interval during which suspension disc vibrates 10 circles by digital milliseco nd meter, then calculate the vibration period. The experiment data is listed on table 1 .

Table 1 experiment data

\begin{tabular}{|c|c|c|c|c|c|c|c|}
\hline$\theta_{0}\left[^{o}\right]$ & $\leq 5$ & 15.0 & 30.0 & 45.0 & 60.0 & 75.0 & 90.0 \\
\hline $\mathrm{T}[\mathrm{S}]$ & 1.352 & 1.360 & 1.376 & 1.406 & 1.450 & 1.504 & 1.570 \\
\hline
\end{tabular}

Table 1 shows that the vibration period of three wire pendulum, which's system factors appeared above, increases with the increase of torsion angle amplitude $\theta_{0}$. We draw the experiment data and 
result of Eq.13 in one diagram, shown in Fig. 6. It is obvious that the experiment data (circle) is very fit to the result of Eq.13 (line). This reveals using Eq.13 to calculate the vibration period of the long cycloid three-wire pendulum is very effective.

\section{Conclusion}

The studies above found that the torsion vibration of the three wire pendulum is nonlinear. The system parameters and initial situation will influence the character of vibration. Theoretical analysis gives the formula of the period which of the long cycloid three-wire pendulum vibrates in any amplitude of torsion angle. The work of this paper enriches the study of vibration of pendulum.

\section{References}

[1] SHENG Zhong-zhi,YI De-wen,YANG Wu'e: College physics Vol.23 No.2 (2004) p.44-46 (in Chinese).

[2] GE Yuhong: Mechanics and Practice Vol.34 No.6 (2012) p.50-54 (in Chinese).

[3] Yanzhu Liu, Liqun Chen: Non-linear Vibration, Higher Education Press, Beijing(2001)(in Chinese).

[4]L.Cveticanin, International Journal of Nonlinear Sciences and Numerical Simulation 10 (2009)1491-1516.

[5] Liu Hui-yin, Essentials of MATLAB R2007, Tsinghua University Press, Beijing (2008) (in Chinese). 